\title{
Metagenomic Analysis of the Fecal Microbiomes of Wild Asian Elephants Reveals Microflora and Enzymes that Mainly Digest Hemicellulose ${ }^{\mathbb{S}}$
}

\author{
Chengbo Zhang ${ }^{1,2}$, Bo $\mathrm{Xu}^{1,2,3,4}$, Zunxi Huang ${ }^{1,2,3,4}$, and Tao $\mathrm{Lu}^{5 *}$ \\ ${ }^{1}$ School of Life Sciences, Yunnan Normal University, Kunming 650500, P.R. China \\ ${ }^{2}$ Engineering Research Center of Sustainable Development and Utilization of Biomass Energy, Ministry of Education, Kunming 650500, P.R. China \\ ${ }^{3}$ Key Laboratory of Yunnan for Biomass Energy and Biotechnology of Environment, Kunming 650500, P.R. China \\ ${ }^{4}$ Key Laboratory of Enzyme Engineering, Yunnan Normal University, Kunming 650500, P.R. China \\ ${ }^{5}$ Yunnan Institute of Microbiology, School of Life Sciences, Yunnan University, 2 North Cui Hu Road, Kunming, Yunnan 650091, P.R. China
}

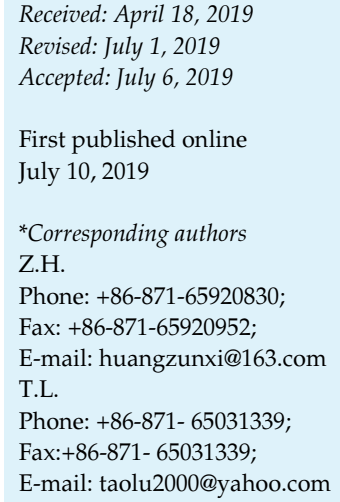

S upplementary data for this paper are available on-line only at http://jmb.or.kr.

pISSN 1017-7825, eISSN 1738-8872

Copyright(C) 2019 by

The Korean Society for Microbiology and Biotechnology
To investigate the diversity of gastrointestinal microflora and lignocellulose-degrading enzymes in wild Asian elephants, three of these animals living in the same group were selected for study from the Wild Elephant Valley in the Xishuangbanna Nature Reserve of Yunnan Province, China. Fresh fecal samples from the three wild Asian elephants were analyzed by metagenomic sequencing to study the diversity of their gastrointestinal microbes and cellulolytic enzymes. There were a high abundance of Firmicutes and a higher abundance of hemicellulose-degrading hydrolases than cellulose-degrading hydrolases in the wild Asian elephants. Furthermore, there were a high abundance and a rich diversity of carbohydrate active enzymes (CAZymes) obtained from the gene set annotation of the three samples, with the majority of them showing low identity with the CAZy database entry. About half of the CAZymes had no species source at the phylum or genus level. These indicated that the wild Asian elephants might possess greater ability to digest hemicellulose than cellulose to provide energy, and moreover, the gastrointestinal tracts of these pachyderms might be a potential source of novel efficient lignocellulose-degrading enzymes. Therefore, the exploitation and utilization of these enzyme resources could help us to alleviate the current energy crisis and ensure food security.

Keywords: Wild asian elephant, metagenome, gastrointestinal tract, hemicellulose, enzyme resource

\section{Introduction}

The efficient utilization of lignocellulose, which makes up the largest biomass on earth, has been a focal point of research all over the world. Settlement of this aporia would help to relieve the global energy crisis and food security problem [1]. Although lignocellulose degradation enzymes have been studied for many years, there are still many unsolved problems such as single-enzyme systems, unclear degradation mechanisms, and low degradation efficiency [2-5]. Thus, the discovery of novel and efficient lignocellulose degradation enzymes and other efficient biomass degradation factors, as well as understanding the biodegradation mechanism of biomass, is important for improving the efficiency of lignocellulose degradation.

Asian elephants (Elephas maximus Linnaeus) are the largest terrestrial herbivorous mammals in Asia [6]. They have a wide-ranging diet that include leaves, branches, stalks, bark, grass and roots [7]. Similar to other herbivorous mammals, such as giant pandas [8], Asian elephants lack homologues of enzymes needed for cellulose degradation [9]. Therefore, the degradation of cellulose in their food 
should depend on their intestinal microflora. Herbivores can get about $70 \%$ of their energy from the decomposition of microbial polysaccharides [9-11]. However, Asian elephants are reported to have a low lignocellulose digestibility $[6,12]$, therefore they must eat a lot of food per day (consuming about 150 kilograms of fresh vegetation a day [13]) to meet their body needs. A high diversity of cellulose-degrading bacteria and Glycoside Hydrolase (GH) family enzymes have been found in Asian elephant feces using metagenomic sequencing analysis, suggesting that Asian elephants have a strong potential for biomass conversion [14]. However, the functional role of gut microbiota in Asian elephants, particularly in cellulose degradation, is still largely unknown.

Our survey showed that the wild Asian elephants in the Wild Elephant Valley in Xishuangbanna, China have a very unique diet. Their main foods are bamboo and wild plantains, which most herbivores choose to avoid. This suggested that the gastrointestinal microbes in the wild Asian elephants might be unique, and might contain novel enzymes for efficiently degrading plant biomass. Herein, in order to explore the diversity of the microbes in wild Asian elephants, and to reveal novel enzymes that are highly effective in degrading lignocellulose contained in their gastrointestinal tract, three wild Asian elephants who live in the same group were selected for study from the Wild Elephant Valley in the Xishuangbanna Nature Reserve of Yunnan Province, China. The fresh fecal samples from the three wild Asian elephants were analyzed by metagenomic sequencing to study the diversity of their gastrointestinal microbes and cellulolytic enzymes.

\section{Materials and Methods}

\section{Ethics Statement}

This study complied with the protocols established by the China Wildlife Conservation Association, as well as the legal requirements of China. An ethics approval from an Animal Care and Use Committee was received as per the local legislation.

\section{Fecal Sample Collection}

Fresh fecal samples from three wild Asian elephants were collected from the Wild Elephant Valley in the Xishuangbanna National Nature Reserve, Yunnan Province, China. The tracks of the wild Asian elephants were followed until they defecated, and the samples were immediately collected from the middle part of the feces. The fresh fecal samples were transported to the laboratory in liquid nitrogen and then stored at $-80^{\circ} \mathrm{C}$ until DNA extraction.

\section{DNA Extraction and Illumina Sequencing}

Genomic DNA were extracted from the fecal samples with the Soil DNA Kit (Omega Bio-Tek, USA) following the protocol provided by the supplier ( $0.25 \mathrm{~g}$ of each fecal sample was used). The quality and quantity of the DNA were determined with a Nanodrop (ND-1000) spectrophotometer (Nanodrop Technologies, USA) and through agarose gel electrophoresis, respectively. DNA samples were stored frozen $\left(-20^{\circ} \mathrm{C}\right)$ until use. The DNA libraries for sequencing were constructed after testing for the qualified samples followed BGI's previous work on human gut microbe metagenomic sequencing [15]. Finally, the qualified DNA libraries were conducted for cluster preparation and sequencing with Illumina HiSeq2500.

\section{Raw Data Processing and de novo Assembly}

Before further analysis, the raw data from Illumina HiSeq2500 sequencing was processed to: (1) remove paired reads with adapter; (2) remove paired reads with base in single-end sequencing reads sequenced to $\mathrm{N}$ ( $\mathrm{N}$ shows unclear base information) over 10\%; (3) remove the paired reads when the number of inferior-quality bases $(\mathrm{sQ}<=5)$ was over $50 \%$ of the total base number of the reads. Then the metagenomic sequence assembling software MEGAHIT [16] (https://github.com/voutcn/megahit) was used to assemble high-quality reads of every sample to obtain longer contigs. Statistics of reads number, length and N50 of the metagenomic sequences were conducted with the local perl script. The highquality reads were blasted against the assembled sequences using SOAP software [17], and the reads utilization of assembly was calculated. According to these statistical data, the effect of assembly could be evaluated.

Open Reading Frames (ORFs) Prediction and Abundance Analysis Prokaryote gene prediction software MetaGeneMark (MGM, version 2.10, default parameters http:/ / exon.gatech.edu/GeneMark/ metagenome/Prediction/) that is suitable for analyzing metagenomes was used to predict ORFs for the assembly results [18], and then collation and statistics of the results were conducted through local perl script. The genes predicted from all the samples were integrated and redundancy removed by using Cd-Hit software. Then the high-quality data obtained from each sample sequencing was compared with the gene sets after removing redundancy (SOAP), and the relative abundance of genes was calculated by comparing the reads number of genes, the length of genes, and the total reads number of samples [19].

\section{Taxonomic Assignment and Functional Classification in Metagenomic Database}

The known sequences from Bacteria, Eukaryota, Archaea, and Viruses in the NT database were first extracted utilizing an internal process to construct a reference database. Blastn was used to blast the nucleotide sequences from the gene group with the constructed NT database. Blastx was used to blast the amino acid 
sequences from the gene group with the constructed NR database. Then according to LCA arithmetic, species annotation was conducted [15]. Based on the gene relative abundance spectrum, a relative abundance spectrum of species at all levels was constructed and statistical analysis was carried out. R software was used to perform two dimensions of sample and species abundance cluster analysis (heatmap) [19]. KRONA software (http://sourceforge. net/projects/krona/) was used to visualize data for species annotation of a single sample [20]. Functional classification of predicted genes was performed by BLAST (version 2.2.21) against the KEGG database [21-23] and the eggNOG (COG) database [24]. CAZymes were searched from the CAZy database $[25,26]$.

\section{PUL Analysis}

Prediction of PUL was carried out following a protocol similar to PULDB [27]. Using the data already generated (comparison of all protein predictions to CAZy), SusC/SusD pairs in all assembled contigs were first identified. Then a simple moving window consisting of five protein predictions upstream and downstream was used to search for proteins with a predicted homologue in the CAZy database. If one was found, the sliding window was moved in that direction, and the search was repeated. The search ended when no more homologues from the CAZy database were found. The PUL were drawn using BioPython [28] and GenomeDiagram [29].

\section{Sequence Data Submission}

Sequence data have been submitted to the NCBI Short Read Archive (SRA) database under accession number PRJNA492972. BioSample accessions: SAMN10141518, SAMN10141519, SAMN10141520.

\section{Results}

\section{Sample Source}

The feces were sampled from three wild Asian elephants from the same living environment with nearly the same diet in the Wild Elephant Valley in Xishuangbanna, Yunnan, China (747-1055 meters above sea level, $22^{\circ} 10^{\prime} 22.68^{\prime \prime} \mathrm{N}$, $\left.100^{\circ} 51^{\prime} 29.39^{\prime \prime} \mathrm{E}\right)$. The Asian elephants were characterized by Asian elephant observers in the protected areas. They were a 4-year-old calf (weaned half a year), a 10-year-old subadult (sexually immature), and a 25-year-old adult (normal). Semi-wild means that the workers in the protected area will provide water for wild Asian elephants at fixed locations, and wild Asian elephants will return to these places to drink and rest after eating in the wild.

\section{Metagenome Sequencing, Assembly, and Annotation}

DNA samples recovered from the 3 Asian elephants' feces were subjected to high throughput sequencing using Illumina Hiseq, and $16.65 \mathrm{~Gb}$ of data were obtained from the 3 samples. With an insert size of $350 \mathrm{bp}, 14.5 \mathrm{~Gb}$ of clean data were obtained after filtering low quality data, and the percentage of clean data was around $87 \%$ (Table S1). The clean data of all 3 samples were assembled, and the average number of contigs was 714,640 , the average genome size was $514,553,566 \mathrm{bp}$, and the average of N50 was $788 \mathrm{bp}$ (Table S2).

Then, the assembly was predicted for an ORF. The ORF sequence of the 3 samples was merged and redundancy was removed after the prediction. The number of genes of non-redundant gene sets was 3,054,637, of which the total length was 1,442.49 $\mathrm{Mbp}$ and the average length was $472.23 \mathrm{bp}$. All non-redundant genes were annotated in 4 databases (NR, COG, KEGG, CAZy), and a total of $4,962,359$ predicted genes was annotated in the 4 databases, accounting for $89.41 \%$ of the total genes. About $85.25 \%$ of the gene annotations were in the NR database, $47.13 \%$ in the COG database, $27.43 \%$ in the KEGG database, and $4.07 \%$ in the CAZy database (Table S3).

\section{Microbial Diversity in the Fecal Metagenomes of the Three Asian Elephants}

The results of the NR annotation were classified. In the sequences of the annotations of all 3 samples, the proportion of bacteria was the highest, with an average accounting for $98 \%$. The average proportion of archaea, fungi, and viruses was $1.06 \%, 0.61 \%, 0.27 \%$, respectively, and there was no significant difference between the 3 samples at the kingdom level. The taxonomic information was further classified by phylum, class, order, family, genus, and species. The highest proportion by phylum was Firmicutes (average $42.9 \%$ ), followed by Bacteroidetes (average 26.8\%), Proteobacteria (average 8.46\%), Fibrobacteres (average $7.68 \%$ ), Spirochaetes (average 5.55\%), and Actinobacteria (average $2.12 \%$ ). The highest proportion by genus was Fibrobacter (average 12.13\%), followed by Treponema (average 7.3\%), Prevotella (average 7.26\%), Bacteroides (average 6.52\%), Bacillus (average 4.95\%), Butyrivibrio (mean 3.76\%), and Ruminococcus (average 2.52\%). These microflorae were directly or indirectly related to lignocellulose degradation. Compared with ruminant animals such as cows [2] and reindeer [30], the gastrointestinal microflorae of wild Asian elephants were significantly diverse, suggesting that the mechanism of lignocellulose degradation by the gastrointestinal microflora in wild Asian elephants might be different.

At both of the phylum and genus levels, it was found that with the increase of wild Asian elephant age, the proportion of Fibrobacteres increased gradually. At the 

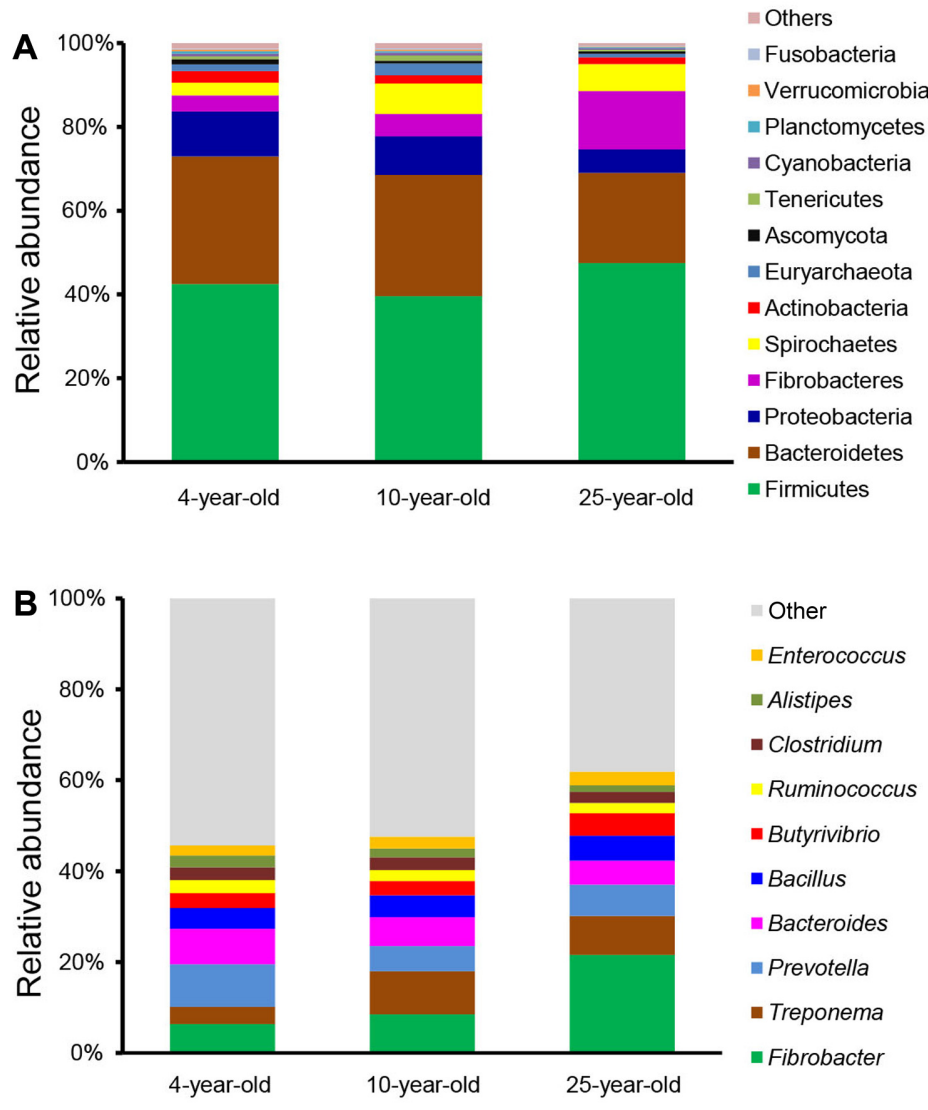

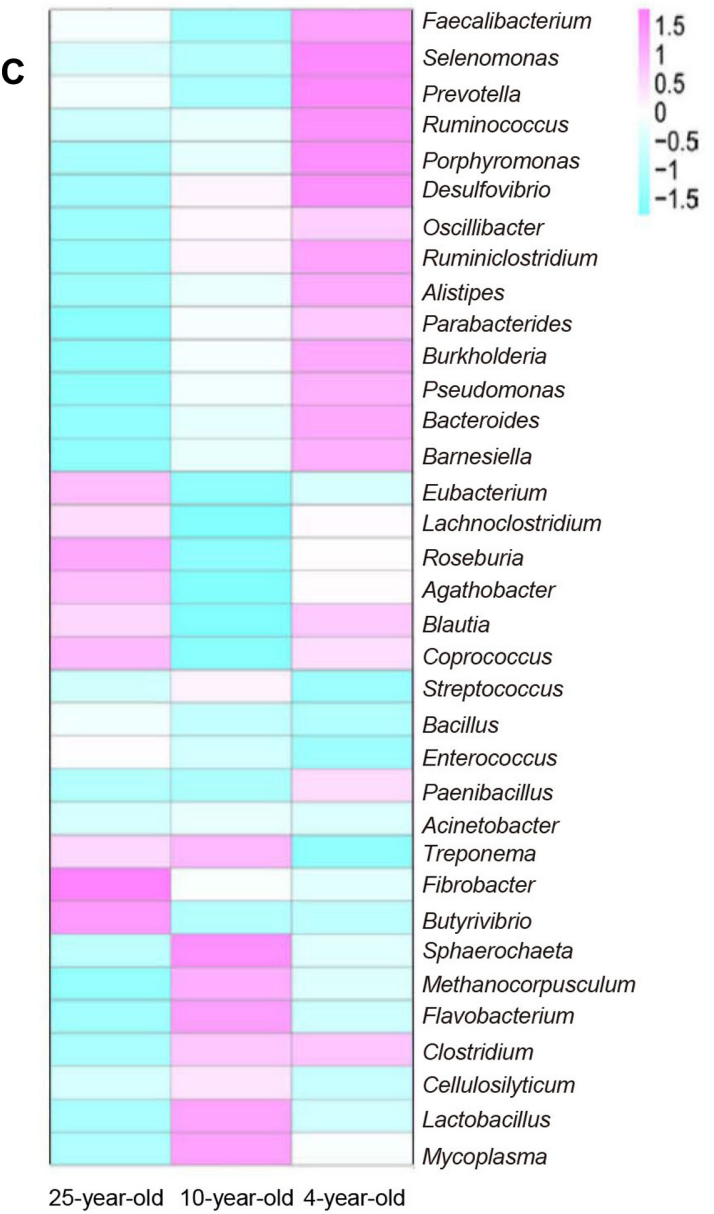

25-year-old 10 -year-old 4-year-old

Fig. 1. (A) Phylogenetic analysis of the three wild Asian elephant fecal samples at phylum level. (B) Relative abundance of different genera in the feces of different age elephants. (C) Based on the species annotation and abundance information of all samples at the genera level, the genus of interest (the top 35 in abundance) were selected and their abundance information in each sample was shown in the heat map.

genus level, the relative abundance of Bacillus also increased with the increasing elephant age (Fig. 1C). In contrast, the relative abundance of Bacteroidetes, Proteobacteria, and Actinobacteria at phylum level (Fig. 1A), as well as Ruminococcus, Ruminiclostridium and Bacteroides et al. at genus level (Figs. 1B and 1C), gradually decreased with the increasing age of the wild Asian elephants. The above microflorae were closely related to the digestion of lignocellulose $[3,31-36]$. The gradually change in abundance of these microflorae might give hints for the subsequent study of the ability of wild Asian elephants to digest lignocellulose as they grow. In addition to the top 10 genera, the relative abundance of other genera was high (Fig. 1B). However, each genus within the group had a very low relative abundance (more than $90 \%$ of them had a relative abundance of less than 0.001, Table S4). Furthermore,

Table 1. Shannon index.

\begin{tabular}{|c|c|c|c|c|c|c|}
\hline Sample name & Phylum & Class & Order & Family & Genus & Species \\
\hline 4-year old & 1.677157 & 2.329577 & 2.667309 & 3.909472 & 4.710428 & 5.837153 \\
\hline 10-year old & 1.776088 & 2.475587 & 2.812012 & 3.862292 & 4.631579 & 5.70086 \\
\hline 25-year old & 1.58855 & 2.108946 & 2.365569 & 3.27689 & 3.901922 & 4.791691 \\
\hline
\end{tabular}


with the growth of the wild Asian elephants, the relative abundance of other genera gradually declined (Fig. 1B), as did the number of genera in the group (Table S4).

The alpha diversity Shannon index (Table 1) was calculated based on the relative abundance of different taxonomic classes of species to evaluate the species diversity of the samples. In the Shannon index results, the 10-year-old wild Asian elephant was higher than others in phylum, class and order, and the 25-year-old wild Asian elephant was the lowest, which indicated that the diversity of higher taxonomic grades may fluctuate in the establishment of microbial flora in the gastrointestinal tract of the wild Asian elephants. From the family level, the Shannon index of the wild Asian elephants gradually decreased with the increasing age of the wild Asian elephants, indicating that the diversity of the microflora decreased with the growth of the wild Asian elephants.

\section{The Repertoire of Carbohydrate Active Enzymes in the} Fecal Metagenomes of the Three Wild Asian Elephants

The ability of the gastrointestinal microbiomes of Asian elephants to convert lignocellulosic materials to shortchain fatty acids (VFAs) depends on their genomic composition of carbohydrate active enzymes (CAZymes). CAZy is a carbohydrate enzyme database, mainly covering Glycoside Hydrolases (GHs), Glycosyl Transferases (GTs), Polysaccharide Lyases (PLs), Carbohydrate Esterases (CEs), and auxiliary enzymes such as Auxiliary Activities (AAs) and Carbohydrate-Binding Modules (CBMs). In the present work, 267,080 genes were annotated to 6 first-class classification and 286 two-level classification of CAZys. Among them, $46.72 \%$ were predicted as novel (with an identity $<40 \%$ to any CAZy database entry), while genes with identities $>80 \%$ to any CAZy database entry were only $1.22 \%$ (Table S5). GHs accounted for $50.93 \%$ of the first-class classification, followed by GTs $(22.87 \%)$, CBMs $(18.71 \%)$, CEs (6.08\%), PLs (1.29\%), and AAs (0.13\%). In the two-level classification, we obtained $117 \mathrm{GH}$ families, 67 GT families, 60 CBM families, $15 \mathrm{CE}$ families, $17 \mathrm{PL}$ families, and 10 AA families, with GT2, GH2, CBM50, GH3 and GT4 being the top 5 CAZymes (Table S6).

Glycoside hydrolases (GHs). The GH enzymes encoded by the three metagenomes in this study fell into 117 families, and $65 \%$ of the identified GHs showed $50 \%$ or lower identity with known proteins, of which GH2 and GH3 were the most prevalent. Eighteen families (GH13, GH43, GH39, GH109, GH31, GH92, GH95, GH78, GH23, GH5, GH94, GH36, GH33, GH20, GH28, GH1, GH73, and GH53) accounted for $50 \%$ of the total sequences (Table S6).
The putative GHs were classified into cellulases, endohemicellulases, xyloglucanases, debranching enzymes, and oligosaccharide degrading enzymes, according to their main substrates and mode of action (Table S7). When compared with CAZymes from the gut metagenomes of other herbivores [2, 14,30,37,38], the wild Asian elephants in this project had a richer diversity of GH family enzymes. The GH families in this study were rich in GH2, GH3, GH5, GH13, GH23, GH31, GH39, GH43, GH78, GH92, GH95 and GH109, which was more diverse than that reported [14]. The GH2, GH3, GH5, and GH43 family genes are also widely found in other intestinal microbial genomes of herbivores such as giant pandas [37], cows [2], reindeer [30], and camels [38]. However, the GH58, GH68, GH75, GH90, GH96, GH100, GH101, GH104, and GH132 found in this work were rarely reported. These families of enzymes are not present in the feces of bovine [2], moose [39] and camel [38], elephant [14], or even biogas fermentaters [40, 41]. Such abundance and diversity of GH family hydrolases in the wild Asian elephant suggested that their gastrointestinal tracts might be sources of novel lignocellulose degrading enzymes.

Carbohydrate-binding modules (CBMs). The CBM domains promote the binding of CAZymes to their carbohydrate substrates, thereby affecting the catalytic activity of the enzymes. The most abundant CBMs that interact with cellulose in the metagenomes of the gut microorganisms in the wild Asian elephants were CBM37, CBM13, CBM2 and CBM6 (Table S6), which was similar to that in camel [38] and bovine rumen [42], and Asian elephants raised in zoos [14]. As for the xylan-bound domain, CBM35, CBM9, CBM54 and CBM4 were abundant, which was also similar to that in the above-mentioned samples, as well as anaerobic biogas fermentation. The proportion of CMB54 was higher than others in this study and was more similar to that in anaerobic biogas fermentation [40]. There were also some less studied CBM families such as CBM51, CBM57, CBM61, CBM62 and CBM66, among which CBM57 accounted for a much larger proportion in the wild Asian elephants than other samples. The rich diversity of CBM families in the wild Asian elephants also suggested that the gastrointestinal tracts of the wild Asian elephants might be potential sources of novel enzymes.

Carbohydrate esterases (CEs), polysaccharide lyases (PLs) and auxiliary activities (AAs). The CEs represent a class of esterases responsible for removing the $-\mathrm{O}$ or $-\mathrm{N}$ ester-bonded modifications to mono-, oligo-, and polysaccharides, facilitating the action of glycosyl hydrolases 
on complex polysaccharides [43]. The CEs predicted in this work belonged to 15 families, of which CE11 and CE1 were the most frequently present in the gene sequences. CE1, CE2, CE3, CE4, CE6, CE7, CE8, and CE12 were relatively rich (Table S6), which were known to play a key role in hemicellulose degradation by enhancing the dissolution of xylan [43]. In addition to CE5, CE13 and CE16, other CE family members were more abundant than that in the camel rumen [38]. The richness and diversity of the CE family enzymes in the wild Asian elephants might be beneficial to the degradation of hemicellulose.

PLs are capable of recognizing uronic acid residues in polysaccharides, catalyzing beta-terminal elimination reactions by releasing sugar residues with unsaturated double bonds at non-reducing ends, ultimately forming unsaturated oligosaccharides. These oligosaccharides often have novel physiological activities while overcoming many of the drawbacks of macromolecular polysaccharides themselves. Therefore, PLs have broad application prospects in scientific research, food industry, medicine and agricultural production. There were 17 families of PLs predicted in this work. Among them, PL1, PL9, and PL11 were the most frequently occurring. Meanwhile, PL6, PL7, PL8, PL10, PL12, PL17, and PL21 accounted for about 20\% (Table S6). PLs were not found in camels and other projects, probably because there is little of them. Thus, the gastrointestinal tracts of the wild Asian elephants have the potential for mining PLs.

The AAs associated with lignin degradation were also abundant in the samples of the wild Asian elephants. AA4 was the most abundant AA family (accounting for 50\%), while that in camel rumen, Asian elephants raised in zoos, and bioreactors AA6 were the most abundant [38]. However, AA6 accounts for only $20 \%$ in this work (Table S6). Since AA4 contains vanillyl-alcohol oxidases (VAO) that catalyze the conversion of a wide range of phenolic compounds bearing side chains at the para-position of the aromatic ring, the ability of the wild Asian elephants to degrade lignin might be higher, and their degrading enzymes might turn out to be special.

\section{Analysis and Comparison of Lignocellulose Degrading Enzymes}

Endoglucanase (E.C. 3.2.1.4), Cellobiohydrolase (E.C. 3.2.1.91), and beta-glucosidase (E.C. 3.2.1.21) were on the annotation of cellulose degradation, corresponding CAZy members were GH1, GH3, GH5, GH6, GH8, GH9, GH12, GH44, GH45, GH48, GH51, GH74, GH116, GH124, and among them the most abundant top 5 were GH3, GH5,
GH1, GH51, and GH9. The enzymes that degrade pectin were annotated with pectinesterase (E.C. 3.1.1.11), endopolygalacturonase (E.C. 3.2.1.15), exopolygalacturonase (E.C. 3.2.1.67), pectate lyase (E.C. 4.2.2.2), exopectate lyase (E.C. 4.2.2.9), and pectin lyase (E.C. 4.2.2.10), corresponding CAZy members were CE8, GH4, GH28, PL1, PL3, PL9, PL10, with CE8 and GH28 being the top 2 families. Endo1,4-beta-D-xylanases (E.C. 3.2.1.8), beta-D-xylosidases (3.2.1.37), acetylxylan esterases (E.C. 3.1.1.72), alpha-Larabinofuranosidases (E.C. 3.2.1.55), alpha-D-glucuronidases (E.C. 3.2.1.139), ferulic acid esterases (E.C. 3.1.1.73), pcoumaric acid esterases (E.C. 3.1.1.-) were on the annotation of hemicellulose degradation, the families of the corresponding CAZys were CE1, CE2, CE3, CE4, CE5, CE6, CE7, CE12, CE13, CE15, GH1, GH2, GH3, GH5, GH8, GH10, GH11, GH30, GH39, GH43, GH51, GH52, GH54, GH62, GH67, GH116, GH120, and the top 5 families were GH2, GH3, GH43, GH39 and GH5. The relative abundance of lignin hydrolases was too low to be shown (Table S8).

\section{Taxonomic Origin of the Predicted CAZymes}

Sequence-based CAZy and NR database annotations suggested that there were 42 phyla of microbes in the taxonomic origin of CAZymes in the fecal samples of the wild Asian elephants. The taxonomic distribution of the predicted CAZymes was shown in Fig. 2. An inspection of the cellulase sequences (families GH1, GH3, GH5, GH9, and GH51, etc.) indicated that the contribution of Firmicutes, Bacteroidetes, and Fibrobacteres in the cellulase repertoire of the wild Asian elephants' metagenomes was 25.8\%, 16.0\%, and $7.3 \%$, respectively (Fig. 2C). At the genus level, the Fibrobacter-encoding cellulase genes were the most abundant (Fig. 2H). This indicated that Fibrobacter was the most important cellulose-degrading bacterium in the wild Asian elephants. An analysis of endohemicellulases (families GH43, GH39, GH5, GH28, and GH53, etc.) revealed that > $45 \%$ of these enzymes were produced by Firmicutes and Bacteroidetes, accounting for more than $75 \%$ of the hemicellulase-matching gene sequences (shown in Fig. 2D), which suggested that the wild Asian elephants might have strong ability to digest hemicellulose. The origins of the genes encoding carbohydrate-debranching enzymes (families $\mathrm{GH} 2, \mathrm{GH} 3, \mathrm{GH} 43$, GH51, and GH78, etc.) were shown in Fig. 2E. A similar distribution was observed with respect to the genes encoding oligosaccharide-degrading enzymes (families GH1, GH3, GH5, GH13, GH31, and GH43, etc.) (Fig. 2F). As for the sequences encoding pectinase (families CE8 and GH28, etc.), they were largely from Firmicutes (23.9\%, Fig. $2 \mathrm{G})$. 


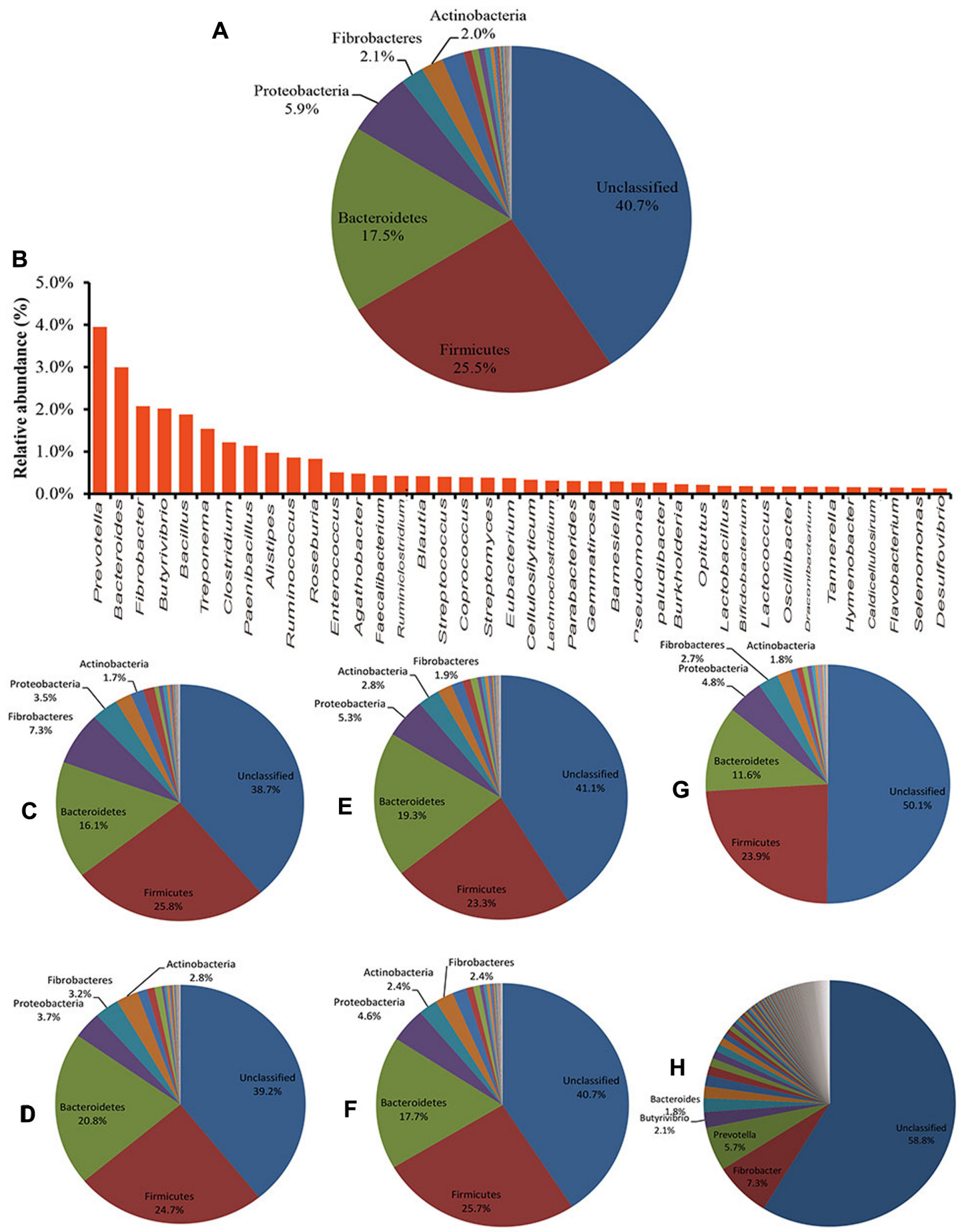

Fig. 2. (A) Phylum level taxonomic distribution of the predicted and unclassified CAZymes; (B) Genus level taxonomic distribution of the $39 \%$ predicted CAZymes. Phylum level taxonomic origin of the predicted and unclassified cellulases (C), endohemicellulases (D), carbohydrate-debranching enzymes (E), oligosaccharide-degrading enzymes (F), and pectinase (G). (H) Genus level taxonomic origin of the predicted cellulases.

The data also showed that $>40 \%$ of the gene sequences that encode CAZymes had no species source at the phylum level, and $>60 \%$ have no species source at the genus level (Figs. 2A and 2B). This implied that there were a large 
number of potentially unknown lignocellulose-degrading enzymes in the gastrointestinal tracts of the wild Asian elephants, which might be the new enzymes we need.

The large contribution of Firmicutes and Bacteroidetes to the coding of CAZymes suggested that in the gastrointestinal tracts of the wild Asian elephants, Firmicutes and Bacteroidetes might work together to degrade lignocellulose, since members of Bacteroides and Firmicutes are known as effective lignocellulose degradants [42]. This was consistent with the fact that the diet of the wild Asian elephants in the wild mainly includes materials with high levels of lignocellulose, such as bamboo and wild plantain.

\section{Discussion}

Asian elephants (Elephas maximus Linnaeus) are the largest terrestrial herbivorous mammals in Asia. They are divided into calves (under 5 years old), subadults (between 5 and 15 years old) and adults (over 15 years old) [44, 45]. The three samples in this study were distributed at different ages. At both of the phylum and genus levels, the relative abundance of their microflorae gradually changed with the age of the wild Asian elephants. Among them, Fibrobacteres and Bacillus were closely related to the digestion of lignocellulose. This suggested that wild Asian elephants might become more capable of digesting lignocellulose as they age. Though analysis of more samples in each age group was needed to statistically test the relationship, it nevertheless provided a hint for the change of ability of wild Asian elephants to digest lignocellulose as they grow.

It is reported that Asian elephants in a German zoo have common dominant bacteria: Firmicutes, Bacteroidetes, Proteobacteria, Fibrobacteres, and Actinobacteria [14]. However, in this study the microbial diversity was more abundant. In addition, there were big differences in gut microbial abundance among wild Asian elephants and other herbivores, such as pandas [37], cows [2], and reindeer [30]. The structure of the gut microbial community in panda is fairly unitary, mainly containing $79 \%$ of Firmicutes and $21 \%$ of Proteobacteria. Cow and reindeer have similar structures of gut microbial community, though Bacteroidetes

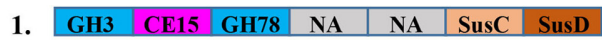

2. GH43 NA

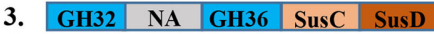

4. GH32 SusC SusD

5. CBM2 CBM4 4 NA 1 SusC SusD

6. GH36 $\quad$ SusC SusD

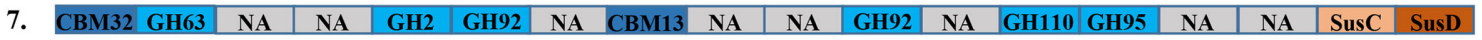

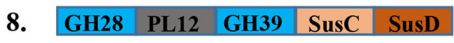

9. \begin{tabular}{|l|l|l|l|l|l|l|}
\hline GT2 & NA & GH3 & NA & NA & SusC & SusD \\
\hline
\end{tabular}

10. GH92

11. CBM13 NA

12. GH51

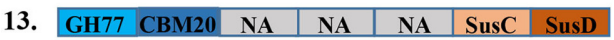

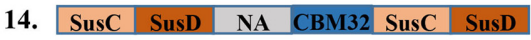

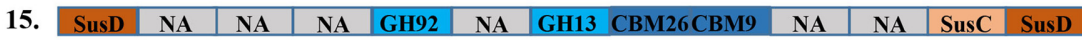

16. GH3 GT49 CE15 SusC SusD

17. CBM50 SusC SusD

Fig. 3. Physical map of selected putative polysaccharide utilization loci (PULs) from the fecal samples of the three wild Asian elephants.

The NA represents other genes of non SusC/SusD/cazy. 
take a larger portion than Firmicutes. From the perspective of rumen and non-ruminal herbivores, the structures of microbial community in Asian elephants and pandas were similar, with Firmicutes occupying a larger proportion than Bacteroidetes. The larger proportion of Firmicutes in panda might be due to the fact that the main food of pandas is bamboo. It is reported that bamboo is more difficult to digest than normal lignocellulose, and giant pandas must have a strong ability to digest hemicellulose to supply energy for their bodies [46]. Thus, we speculated that Asian elephants, like giant pandas, might digest hemicelluloses more efficiently than digest cellulose.
Accordingly, the diversity and abundance of hemicellulosedegrading related hydrolases in the wild Asian elephants of all ages were higher than that of cellulose-degrading related hydrolases (Table S8). The CAZy data combined with Sus-like genes (SusC or SusD) clustering revealed 17 identity groups (polysaccharide utilization loci, PULs) in this study, which encodes all the components required for binding, transportation, and depolymerization of a specific sugar chain structure. Similar to the analysis of novel polysaccharide utilization loci in Castor canadensis fecal microbiomes [47], in this study a majority of the enzymes (GH3, GH36, GH39, GH43, GH63, GH92, GH95, GH110,
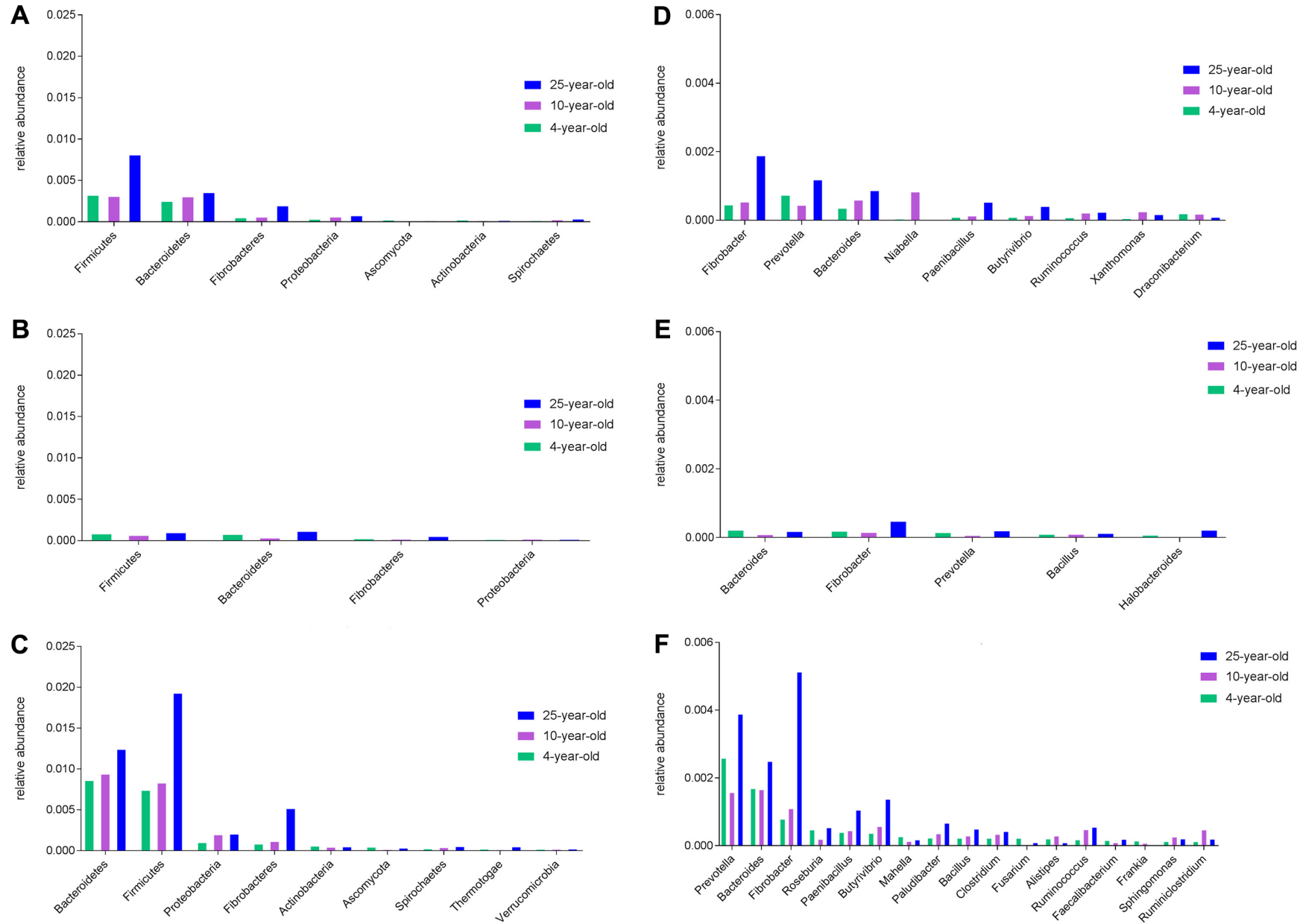

Fig. 4. The abundance of phylum- and genus-level microbial communities corresponding to hydrolytic enzymes that degrade cellulose, pectin and hemicellulose in the 3 fecal samples.

(A) The abundance of phylum-level microbial communities corresponding to hydrolytic enzymes that degrade cellulose. (B) The abundance of phylum-level microbial communities corresponding to hydrolytic enzymes that degrade pectin. (C) The abundance of phylum-level microbial communities corresponding to hydrolytic enzymes that degrade hemicellulose. (D) The abundance of genus-level microbial communities corresponding to hydrolytic enzymes that degrade cellulose. (E) The abundance of genus-level microbial communities corresponding to hydrolytic enzymes that degrade pectin. (F) The abundance of genus-level microbial communities corresponding to hydrolytic enzymes that degrade hemicellulose. 
CE15 etc.) in PULs targeted hemicellulose degradation, only GH9 was responsible for cellulose degradation, GH28 and GH32 mainly degraded pectin (Fig. 3). These implied that wild Asian elephants might mainly digest hemicellulose to supply energy.

Furthermore, at both the phylum and genus levels, by comparing the abundance and diversity of the corresponding bacteria of hemicellulose-degrading enzymes with those of cellulose-degrading enzymes, it was not difficult to find that the abundance and diversity of hemicellulosedegrading bacteria were very high (Fig. 4), which also implied that wild Asian elephants might provide more energy for their bodies by degrading hemicellulose than by degrading cellulose.

Finally, the repertoire of carbohydrate active enzymes and taxonomic origin of the predicted CAZymes in the fecal metagenomes of the three wild Asian elephants showed low identity with the CAZy database entry, and about half of the CAZymes had no species source at the phylum and genus level. This suggested that the wild Asian elephant gastrointestinal tracts might contain a large amount of novel lignocellulose-degrading enzymes, which could be a potential source of novel efficient lignocellulosedegrading enzymes. The exploitation and utilization of these enzyme resources could help us to alleviate the current energy crisis and ensure food security.

\section{Acknowledgments}

This study was supported by the National Key Research and Development Program of China (Grant No. 2017YFB0308401); the National Natural Science Foundation of China (Grant No. 31660304, No. 31560305 and No. 31860299); and Applied Basic Research Foundation of Yunnan Province (Grant No. 201401PC00224).

\section{Conflict of Interest}

The authors have no financial conflicts of interest to declare.

\section{References}

1. Lynd LR, Laser MS, Bransby D, Dale BE, Davison B, Hamilton R, et al. 2008. How biotech can transform biofuels. Nat. Biotechnol. 26:169-172.

2. Hess M, Sczyrba A, Egan R, Kim TW, Chokhawala H, Schroth G, et al. 2011. Metagenomic discovery of biomassdegrading genes and genomes from cow rumen. Science 331: 463-467.
3. Wang C, Dong D, Wang H, Muller K, Qin Y, Wang H, et al. 2016. Metagenomic analysis of microbial consortia enriched from compost: new insights into the role of Actinobacteria in lignocellulose decomposition. Biotechnol. Biofuels 9: 22.

4. Jose VL, More RP, Appoothy T, Arun AS. 2017. In depth analysis of rumen microbial and carbohydrate-active enzymes profile in Indian crossbred cattle. Syst. Appl. Microbiol. 40: 160-170.

5. Mosier N, Wyman C, Dale B, Elander R, Lee YY, Holtzapple M, et al. 2005. Features of promising technologies for pretreatment of lignocellulosic biomass. Bioresour. Technol. 96: 673-686.

6. Shoshani J EJ. 1982. Elephas maximus. Mammalian Species 182: 1-8.

7. Shoshani J EJ. 1982. Elephas maximus. Mammalian Species 182: 42-51.

8. Li R, Fan W, Tian G, Zhu H, He L, Cai J, et al. 2010. The sequence and de novo assembly of the giant panda genome. Nature 463: 311-317.

9. Conklin-Brittain NL. 1995. The digestive system in mammals. Food, form and function. Int. J. Primatol. 16: 699-701.

10. Flint HJ, Bayer EA, Rincon MT, Lamed R, White BA. 2008. Polysaccharide utilization by gut bacteria: potential for new insights from genomic analysis. Nat. Rev. Microbiol. 6: 121131.

11. Flint HJ, Scott KP, Duncan SH, Louis P, Forano E. 2012. Microbial degradation of complex carbohydrates in the gut. Gut Microbes 3: 289-306.

12. Shoshani J EJ. 1982. Elephas maximus. Mammalian Species 182: 78-79.

13. Samansiri KAP, Weerakoon DK. 2006. Feeding Behaviour of Asian Elephants in the Northwestern Region of Sri Lanka. Gajah.

14. Ilmberger N, Gullert S, Dannenberg J, Rabausch U, Torres J, Wemheuer B, et al. 2014. A comparative metagenome survey of the fecal microbiota of a breast- and a plant-fed Asian elephant reveals an unexpectedly high diversity of glycoside hydrolase family enzymes. PLoS One 9: e106707.

15. Qin J, Li R, Raes J, Arumugam M, Burgdorf KS, Manichanh C, et al. 2010. A human gut microbial gene catalogue established by metagenomic sequencing. Nature 464: 59-65.

16. Li D, Liu CM, Luo R, Sadakane K, Lam TW. 2015. MEGAHIT: an ultra-fast single-node solution for large and complex metagenomics assembly via succinct de Bruijn graph. Bioinformatics 31: 1674-1676.

17. Li R, Li Y, Kristiansen K, Wang J. 2008. SOAP: short oligonucleotide alignment program. Bioinformatics 24: 713-714.

18. Zhu W, Lomsadze A, Borodovsky M. 2010. Ab initio gene identification in metagenomic sequences. Nucleic Acids Res. 38(12): e132.

19. Qin J, Li Y, Cai Z, Li S, Zhu J, Zhang F, et al. 2012. A metagenome-wide association study of gut microbiota in type 2 diabetes. Nature 490: 55-60. 
20. Ondov BD, Bergman NH, Phillippy AM. 2011. Interactive metagenomic visualization in a Web browser. BMC Bioinformatics 12: 385.

21. Kanehisa M, Goto S, Kawashima S, Okuno Y, Hattori M. 2004. The KEGG resource for deciphering the genome. Nucleic Acids Res. 32: D277-D280.

22. Kanehisa M. 1997. A database for post-genome analysis. Trends Genet. 13: 375-376.

23. Kanehisa M, Goto S, Hattori M, Aoki-Kinoshita KF, Itoh M, Kawashima S, et al. 2006. From genomics to chemical genomics: new developments in KEGG. Nucleic Acids Res. 34: D354-D357.

24. Powell S, Szklarczyk D, Trachana K, Roth A, Kuhn M, Muller J, et al. 2012. eggNOG v3.0: orthologous groups covering 1133 organisms at 41 different taxonomic ranges. Nucleic Acids Res. 40: D284-D289.

25. Drula E, Golaconda Ramulu H, Coutinho PM, Lombard V, Henrissat B. 2013. The carbohydrate-active enzymes database (CAZy) in 2013. Nucleic Acids Res. 42: D490-D495.

26. Cantarel BL, Rancurel C, Coutinho PM, Bernard T, Lombard V, Henrissat B. 2008. The Carbohydrate-Active EnZymes database (CAZy): an expert resource for Glycogenomics. Nucleic Acids Res. 37: D233-D238.

27. Terrapon N, Lombard V, Gilbert HJ, Henrissat B. 2015. Automatic prediction of polysaccharide utilization loci in Bacteroidetes species. Bioinformatics 31: 647-655.

28. Cock PJ, Antao T, Chang JT, Chapman BA, Cox CJ, Dalke A, et al. 2009. Biopython: freely available Python tools for computational molecular biology and bioinformatics. Bioinformatics 25: 1422-1423.

29. Pritchard L, White JA, Birch PR, Toth IK. 2006. GenomeDiagram: a python package for the visualization of large-scale genomic data. Bioinformatics 22: 616-617.

30. Pope PB, Mackenzie AK, Gregor I, Smith W, Sundset MA, McHardy AC, et al. 2012. Metagenomics of the Svalbard reindeer rumen microbiome reveals abundance of polysaccharide utilization loci. PLoS One 7: e38571.

31. Stahl DA, Flesher B, Mansfield HR, Montgomery L. 1988. Use of phylogenetically based hybridization probes for studies of ruminal microbial ecology. Appl. Environ. Microbiol. 54: 1079-1084.

32. Abbott DW, Boraston AB. 2008. Structural biology of pectin degradation by Enterobacteriaceae. Microbiol. Mol. Biol. Rev. 72: 301-316.

33. Reddy AP, Simmons CW, D'Haeseleer P, Khudyakov J, Burd H, Hadi M, et al. 2013. Discovery of microorganisms and enzymes involved in high-solids decomposition of rice straw using metagenomic analyses. PLoS One 8: e77985.

34. Saoudi B, Habbeche A, Kerouaz B, Haberra S, Romdhane $\mathrm{ZB}$, Tichati L, et al. 2015. Purification and characterization of a new thermoalkaliphilic pectate lyase from Actinomadura keratinilytica Cpt20. Process Biochem. 50: 2259-2266.
35. Palevich N, Kelly WJ, Leahy SC, Altermann E, Rakonjac J, Attwood GT. 2017. The complete genome sequence of the rumen bacterium Butyrivibrio hungatei MB2003. Stand. Genomic Sci. 12: 72.

36. Farro E, Leite A, Silva IA, Filgueiras JG, de Azevedo ER, Polikarpov I, et al. 2018. GH43 endo-arabinanase from Bacillus licheniformis: Structure, activity and unexpected synergistic effect on cellulose enzymatic hydrolysis. Int. J. Biol. Macromol. 117: 7-16.

37. Zhu L, Wu Q, Dai J, Zhang S, Wei F. 2011. Evidence of cellulose metabolism by the giant panda gut microbiome. Proc. Natl. Acad. Sci. USA 108: 17714-17719.

38. Gharechahi J, Salekdeh GH. 2018. A metagenomic analysis of the camel rumen's microbiome identifies the major microbes responsible for lignocellulose degradation and fermentation. Biotechnol. Biofuels 11: 216.

39. Svartstrom O, Alneberg J, Terrapon N, Lombard V, de Bruijn I, Malmsten J, et al. 2017. Ninety-nine de novo assembled genomes from the moose (Alces alces) rumen microbiome provide new insights into microbial plant biomass degradation. ISME J. 11: 2538-2551.

40. Gullert S, Fischer MA, Turaev D, Noebauer B, Ilmberger N, Wemheuer B, et al. 2016. Deep metagenome and metatranscriptome analyses of microbial communities affiliated with an industrial biogas fermenter, a cow rumen, and elephant feces reveal major differences in carbohydrate hydrolysis strategies. Biotechnol. Biofuels 9: 121.

41. Campanaro S, Treu L, Kougias PG, Francisci DD, Valle G, Angelidaki I. 2016. Metagenomic analysis and functional characterization of the biogas microbiome using high throughput shotgun sequencing and a novel binning strategy. Biotechnol. Biofuels 9: 1-17.

42. Jami E, Israel A, Kotser A, Mizrahi I. 2013. Exploring the bovine rumen bacterial community from birth to adulthood. ISME J. 7: 1069-1079.

43. Armendariz-Ruiz M, Rodriguez-Gonzalez JA, Camacho-Ruiz RM, Mateos-Diaz JC. 2018. Carbohydrate Esterases: An Overview. Methods Mol. Biol. 1835: 39-68.

44. Reilly J. 2010. Growth in the Sumatran elephant (Elephas maximus sumatranus) and age estimation based on dung diameter. Proceedings of the Zoological Society of London 258: 205-213.

45. Sukumar R. 2006. A brief review of the status, distribution and biology of wild Asian elephants Elephas maximus. Int. Zoo Yearbook 40: 1-8.

46. Zhang W, Liu W, Hou R, Zhang L, Schmitz-Esser S, Sun H, et al. 2018. Age-associated microbiome shows the giant panda lives on hemicelluloses, not on cellulose. ISME J. 12: 1319-1328.

47. Armstrong Z, Mewis K, Liu F, Morgan-Lang C, Scofield M, Durno E, et al. 2018. Metagenomics reveals functional synergy and novel polysaccharide utilization loci in the Castor canadensis fecal microbiome. ISME J. 12: 2757-2769. 